\title{
Distinct patterns of inflammation in the airway lumen and bronchial mucosa of children with cystic fibrosis
}

\author{
Nicolas Regamey, ${ }^{1,2,3}$ Lemonia Tsartsali, ${ }^{1}$ Tom N Hilliard, ${ }^{1,2}$ Oliver Fuchs, ${ }^{3}$ \\ Hui-Leng Tan, ${ }^{1,2}$ Jie Zhu, ${ }^{2}$ Yu-Sheng Qiu, ${ }^{2}$ Eric W F W Alton, ${ }^{2}$ Peter $\mathrm{K}$ Jeffery, ${ }^{2}$ \\ Andrew Bush, ${ }^{1}$ Jane C Davies ${ }^{1,2}$
}

\begin{abstract}
- Additional materials are published online only. To view these files please visit the journal online (http://thorax.bmj.com/ content/67/2.toc)

1 Department of Paediatric Respiratory Medicine, Royal Brompton Hospital, London, UK ${ }^{2}$ Department of Gene Therapy, National Heart and Lung Institute, Imperial College London, London, UK ${ }^{3}$ Division of Paediatric Respiratory Medicine, Department of Paediatrics, University Hospital of Bern, Bern, Switzerland
\end{abstract}

\section{Correspondence to} Nicolas Regamey, Division of Respiratory Medicine, Department of Paediatrics, University Hospital of Bern, 3010 Inselspital, Bern,

Switzerland;

nicolas.regamey@insel.ch

Received 8 June 2011 Accepted 21 September 2011 Published Online First 18 October 2011

\begin{abstract}
Background Studies in cystic fibrosis (CF) generally focus on inflammation present in the airway lumen. Little is known about inflammation occurring in the airway wall, the site ultimately destroyed in end-stage disease. Objective To test the hypothesis that inflammatory patterns in the lumen do not reflect those in the airway wall of children with CF.

Methods Bronchoalveolar lavage (BAL) fluid and endobronchial biopsies were obtained from 46 children with CF and 16 disease-free controls. BAL cell differential was assessed using May-Gruenwald-stained cytospins. Area profile counts of bronchial tissue immunopositive inflammatory cells were determined.
\end{abstract}

Results BAL fluid from children with CF had a predominance of neutrophils compared with controls (median $810 \times 10^{3} / \mathrm{ml}$ vs $1 \times 10^{3} / \mathrm{ml}, \mathrm{p}<0.0001$ ). In contrast, subepithelial bronchial tissue from children with CF was characterised by a predominance of lymphocytes (median 961 vs 717 cells $/ \mathrm{mm}^{2}, \mathrm{p}=0.014$ ), of which $82 \%$ were (CD3) T lymphocytes. In chest exacerbations, BAL fluid from children with CF had more inflammatory cells of all types compared with those with stable disease whereas, in biopsies, only the numbers of lymphocytes and macrophages, but not of neutrophils, were higher. A positive culture of Pseudomonas aeruginosa was associated with higher numbers of $T$ lymphocytes in subepithelial bronchial tissue (median 1174 vs 714 cells/ $\mathrm{mm}^{2}, p=0.029$ ), but no changes were seen in BAL fluid. Cell counts in BAL fluid and biopsies were positively correlated with age but were unrelated to each other.

Conclusion The inflammatory response in the $\mathrm{CF}$ airway is compartmentalised. In contrast to the neutrophildominated inflammation present in the airway lumen, the bronchial mucosa is characterised by the recruitment and accumulation of lymphocytes.

\section{INTRODUCTION}

Pulmonary disease is the most significant cause of morbidity and mortality in cystic fibrosis (CF). ${ }^{1} \mathrm{~A}$ hallmark of CF lung disease is chronic bacterial infection and the predominance of large numbers of neutrophils in the airway lumen. Neutrophilswhich release an array of mediators, oxidants and proteases, including neutrophil elastase-are thus considered to play a major role in ensuing tissue damage and CF disease progression. In contrast to the large number of studies that have used bronchoalveolar lavage (BAL) to focus on the lumen, the CF airway wall has been underinvestigated. Indeed,

\section{Key messages}

What is the key question?

- Do inflammatory patterns in the airway lumen of children with cystic fibrosis (CF) reflect those in the airway wall, the site ultimately destroyed in end-stage disease?

What is the bottom line?

- The inflammatory patterns and responses to infective stimuli in the airway lumen and the airway wall of children with $\mathrm{CF}$ are distinct, and thus bronchoalveolar lavage and endobronchial biopsy provide different but complementary information.

Why read on?

- This paper provides a detailed description of inflammatory cellular processes in the airways of subjects with $\mathrm{CF}$ in early disease stages.

little is known about the nature of inflammation in the airway wall, particularly that which occurs in the bronchial mucosa.

Studies in asthma have shown poor agreement between the patterns of inflammation in the airway lumen and that occurring in the bronchial mucosa ${ }^{2}$ suggesting that BAL and endobronchial biopsy measure dissimilar patterns of inflammation in these distinct compartments. In the few studies that have assessed airway wall inflammation in CF, the predominance of neutrophils seen in BAL fluid was not present in the bronchial wall. ${ }^{3-5}$ Quantitative assessment of inflammatory cells in the bronchial mucosa has shown an accumulation of lymphocytes, especially at the distal level where intense tissue damage is observed. In contrast, neutrophils appeared to accumulate preferentially in the surface epithelium, suggesting migration of these cells towards the airway lumen. ${ }^{4}$ As these studies have used end-stage CF lung tissue obtained at autopsy or transplantation, data on the pattern of inflammation in the bronchial mucosa in earlier stages of CF lung disease are lacking.

Airway disease is present early in life in CF, even in asymptomatic infants. Infection and inflammation can be detected in the BAL fluid of infants with CF even at a few weeks of age. ${ }^{6}$ Structural airway wall changes, referred to as airway remodelling, also begin early in life in CF. CT scanning demonstrates the 
presence of thickened airway walls, narrowed airway lumina, air trapping and bronchiectasis in young children and infants, ${ }^{7-9}$ and lung function has been shown to be diminished in infants with $\mathrm{CF}^{10}{ }^{11}$ However, the sequence of inflammation and remodelling events in the disease process is poorly understood. ${ }^{12}$ Airway remodelling seems, at least in part, to be unrelated to airway lumen inflammation, ${ }^{13} 14$ but its relationship to the inflammatory infiltrate in the bronchial mucosa has not been explored. Endobronchial studies during early stages of CF lung disease could assess to what extent-if at all-the patterns of inflammation in the airway lumen reflect airway wall changes, shed light on pathophysiological processes occurring in the airway wall and possibly allow assessment of longitudinal changes.

Based on the findings in asthma which show that differing yet complementary information is gleaned from BAL and endobronchial biopsy, we hypothesised that the CF airway inflammatory processes would be compartmentalised and that the luminal patterns of inflammation would not reflect changes in the bronchial mucosa. We tested this hypothesis using BAL fluid and endobronchial biopsies obtained from children with CF and disease-free controls.

\section{METHODS \\ Subjects}

Subjects were prospectively recruited between March 2003 and June 2007 from children with CF diagnosed using standard criteria $^{15}$ who were undergoing bronchoscopy for a clinical indication and control children who had been referred to a tertiary centre, but in whom no lower airway disease was found upon investigations that included clinically indicated flexible bronchoscopy. Specifically, inclusion criteria for control children were: (1) no prior history of lower airway disease; (2) no medication taken; (3) no macroscopic signs of inflammation seen on bronchoscopy (ie, no abnormal secretion, mucosal oedema or redness); (4) no viral detection or growth of pathogens in BAL fluid; and (5) BAL fluid with normal differential cell counts (ie, $>80 \%$ macrophages). ${ }^{16}$ One control was a child undergoing cardiac catheterisation where informed consent was given to perform bronchoscopy for research reasons. Exclusion criteria for both subject groups were bleeding tendency or treatment with anticoagulants and preterm birth before 36 weeks of gestation. Data from some of the children included in the present study have been previously reported. ${ }^{13}{ }^{17-20}$ The study was approved by the Royal Brompton Harefield and NHLI ethics committee. Informed consent and age-appropriate assent was obtained from parents and children respectively.

\section{Flexible bronchoscopy}

Bronchoscopy was performed as previously described under general anaesthesia. ${ }^{18}$ BAL was performed using three aliquots of $1 \mathrm{ml} / \mathrm{kg} 0.9 \%$ saline at room temperature instilled separately into one or two lobes (usually the right middle lobe and the clinically most affected lobe), and the returns pooled. Up to five endobronchial biopsies (median 3, range 1-5) were taken from a standardised site (subsegmental bronchi of the right lower lobe) and processed into paraffin wax blocks as described previously. ${ }^{18}$ In order to be included in the study, each child was a priori required to have at least one evaluable biopsy ${ }^{18}$ with at least $0.1 \mathrm{~mm}^{2}$ of subepithelial tissue. ${ }^{21}$

\section{BAL fluid analysis}

BAL fluid was processed as described previously. ${ }^{13}$ Viable cells were counted and cell differentials were assessed on cytospin preparations using May-Gruenwald-Giemsa staining. Bacterial and fungal growth was assessed and viruses (respiratory syncytial virus, parainfluenza 1,2 and 3, influenza A and B, adenovirus and cytomegalovirus) were sought using direct immunofluorescence and/or rapid viral tissue culture.

\section{Immunohistochemistry}

Endobronchial biopsies were immmunostained for neutrophils (neutrophil elastase), T lymphocytes (CD3) and B lymphocytes (CD20) lymphocytes, macrophages (CD68), eosinophils (EG2) and mast cells (tryptase). Area profile counts of immunopositive cells in subepithelial tissue were performed by one of two investigators (NR and LT) blinded to the study groups. ${ }^{22}$

\section{Tissue morphometry}

Morphometry was performed on $3 \mu \mathrm{m}$ thick haematoxylin and eosin-stained biopsy sections as described previously. ${ }^{13}{ }^{19}$ Reticular basement membrane (RBM) thickness was measured on coded sections by taking the geometric mean of 40 measurements at $20 \mu \mathrm{m}$ intervals. ${ }^{19} 23$ The airway smooth muscle (ASM) volume fraction was measured, applying point and line intersection counting using previously recommended and validated methods of design-based stereology. ${ }^{19}$

\section{Power calculation}

Biopsy data from a study in atopic adults indicate that a sample size between 13 and 48 subjects is needed to detect at least one doubling difference in cell number per $0.1 \mathrm{~mm}^{2}$ for a particular inflammatory cell type in a study using a parallel design such as ours, with $\alpha=0.05$ and power of $0.80 .^{24}$

\section{Statistical analysis}

Categorical data were examined using a $\chi^{2}$ test. For normally distributed data, shown as mean (SD), between-group comparisons were performed with Student $t$ tests and associations tested by Pearson correlation. ${ }^{25}$ For non-normally distributed data, shown as median (range or IOR), between-group comparisons were performed with Mann-Whitney U-tests and associations tested by Spearman rank correlation. Multivariable linear regression models were used to adjust for possible confounders. Additional details are provided in the online supplement.

\section{RESULTS}

\section{Patients}

The study included 62 children of median age 7.3 years (range 0.2-16.8). Forty-six children had CF and underwent bronchoscopy for a clinical indication, and there were 16 controls without lower airway disease (table 1).

\section{Neutrophilic inflammation in BAL fluid}

Inflammatory cell counts in BAL fluid were available in 33 (72\%) of the children with CF and in 10 (63\%) controls, and cell differentials were available in $35(76 \%)$ of the CF children and in $12(75 \%)$ controls.

BAL fluid from children with CF contained more inflammatory cells and more of every cell type compared with controls (table 2). There was a predominance of neutrophils and macrophages, with moderate numbers of lymphocytes (figure 1A). In contrast, most of the inflammatory cells in control BAL fluid were macrophages. Mast cells were rarely seen in either CF or control BAL fluid (data not shown).

\section{Biopsy analysis}

A total of 181 biopsies were taken from the 62 children; 121 $(67 \%)$ of these biopsies were considered evaluable, according to 
Table 1 Subject characteristics $(n=62)$

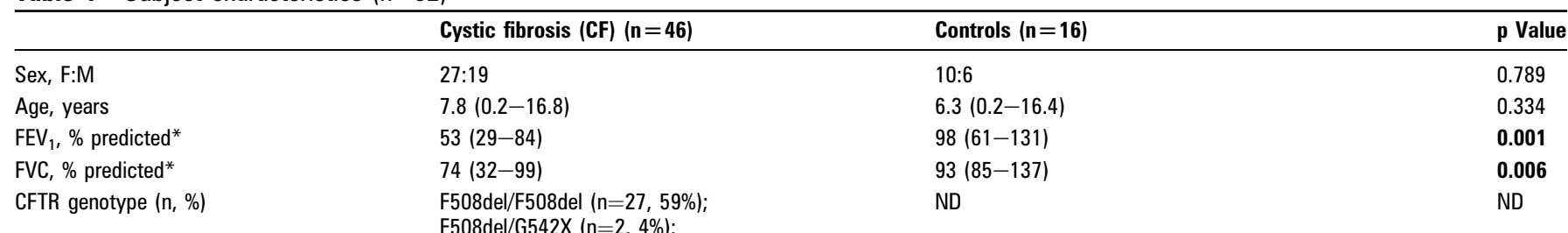

Indication for bronchoscopy (n)

Pathogens in BAL fluid

\author{
Viruses: \\ Parainfluenza virus $3(n=2 ; 4 \%)$ \\ Bacteria: \\ Pseudomonas aeruginosa $(\mathrm{n}=11 ; 24 \%)$ \\ Other $(n=6 ; 13 \%) \neq$ \\ Fungi: \\ Aspergillus ( $\mathrm{n}=13 ; 28 \%) \S$ \\ Candida albicans $(\mathrm{n}=4 ; 9 \%)$
}

Values expressed as median (range) unless otherwise indicated.

*Data available for 29 children with CF and six control children.

tChest exacerbation based on the decision to hospitalise and treat with intravenous antibiotics. ${ }^{26}$

$\ddagger$ Staphylococcus aureus $(n=2)$, Haemophilus influenzae $(n=1)$, Mycobacterium chelonae $(n=2)$, Stenotrophomonas maltophilia $(n=1)$.

$\S$ Aspergillus fumigatus $(\mathrm{n}=12)$, Aspergillus niger $(\mathrm{n}=1)$.

$\mathrm{BAL}$, bronchoalveolar lavage; $\mathrm{FEV}_{1}$, forced expiratory volume in $1 \mathrm{~s}$; FVC, forced vital capacity; ND, not determined.

predefined criteria, ${ }^{18}$ and had at least $0.1 \mathrm{~mm}^{2}$ of subepithelial tissue. $^{21}$ Twenty-eight (45\%) children had one evaluable biopsy, $16(26 \%)$ had two, 13 (21\%) had three, $3(5 \%)$ had four and 2 (3\%) had five. The median number of biopsies analysed per child was similar in the disease groups (2 (range 1-3) for CF and 1 (range $1-3$ ) for controls, $\mathrm{p}=0.601$ ), as was the median area of subepithelial tissue assessed $(0.42$ (range $0.16-0.76) \mathrm{mm}^{2}$ for CF and 0.15 (range $0.13-0.45$ ) $\mathrm{mm}^{2}$ for controls, $\mathrm{p}=0.152$ ).

\section{Lymphocytic inflammation in bronchial mucosa}

There were more subepithelial inflammatory cells in CF tissue than in control tissue, with a similar cell distribution in both groups (table 2). CF subepithelial tissue was thus characterised by an infiltrate predominantly consisting of lymphocytes (median 961 cells $/ \mathrm{mm}^{2}$ vs 717 cells $/ \mathrm{mm}^{2}, \mathrm{p}=0.014$ ) and macrophages, with few neutrophils, eosinophils or mast cells (figure 1B). The lymphocytic infiltrate consisted mainly of $\mathrm{T}$ lymphocytes both in CF tissue (82\% T lymphocytes vs 18\% B lymphocytes) and in control tissue (87\% T lymphocytes vs 13\% B lymphocytes).

Neutrophil, lymphocyte, macrophage and mast cell counts in BAL fluid were not correlated with counts for the same cell types in the biopsies. There was a weak relationship between eosinophil counts in BAL fluid and in subepithelial tissue within the CF group ( $r=0.43, p=0.019)$.

\section{Relationship of airway inflammation with age, pulmonary function tests and CFTR genotype}

Within the CF group, the number of BAL inflammatory cells $(r=0.68, p<0.0001)$, neutrophils $(r=0.64, p<0.0001)$, macrophages $(\mathrm{r}=0.44, \mathrm{p}=0.011)$ and eosinophils $(\mathrm{r}=0.64, \mathrm{p}<0.0001)$ correlated positively with age. In contrast, in the bronchial mucosa, only macrophage counts were positively related to age $(r=0.32$, $\mathrm{p}=0.042$ ).

In the 29 children old enough to perform reliable spirometry, inflammatory cell counts in BAL fluid or biopsies were not related to any test value of pulmonary function (forced expiratory volume in $1 \mathrm{~s}\left(\mathrm{FEV}_{1}\right)$, forced vital capacity (FVC) or $\mathrm{FEV}_{1} / \mathrm{FVC}$ ). There was no relationship between CFTR genotype and any inflammatory cell counts in BAL fluid or biopsies.

\section{Different patterns of inflammation during chest exacerbations and phases of clinical stability in BAL fluid and biopsies}

Bronchoscopic sampling had been performed during chest exacerbations-defined in terms of the decision to hospitalise and treat with intravenous antibiotics ${ }^{26}$-in 36 (78\%) of the children with CF. The other 10 children underwent the procedure while well and did not require intravenous antibiotics either before or after the procedure. All inflammatory cell types in BAL fluid were higher in children investigated during chest exacerbations than in those who were well (table 3). These differences remained significant after adjustment for age for all cell types except for eosinophils. In contrast, in bronchial subepithelial tissue, only $\mathrm{T}$ lymphocytes and macrophages were significantly higher during chest exacerbations compared with those who were well. These differences remained significant after adjustment for age. 
Table 2 Total and differential inflammatory cell counts in bronchoalveolar lavage (BAL) fluid and in subepithelial bronchial tissue obtained from children with cystic fibrosis (CF) $(n=46)$ and controls $(n=16)$

\begin{tabular}{clll}
\hline & CF & Controls & p Value \\
\hline BAL (cells $\times 10^{3}$ per ml) & & & \\
Total inflammatory cells & $1382(753-2951)$ & $102(78-190)$ & $<\mathbf{0 . 0 0 0 1}$ \\
$\quad$ Neutrophils & $810(285-1230), 49 \%$ & $1(0-6), 1 \%$ & $<\mathbf{0 . 0 0 1}$ \\
Lymphocytes & $37(11-126), 2 \%$ & $3(1-5), 2 \%$ & $<\mathbf{0 . 0 0 1}$ \\
Macrophages & $641(329-1042), 48 \%$ & $96(73-168), 97 \%$ & $<0.0001$ \\
Eosinophils & $11(1-41), 1 \%$ & $0(0-1), 0 \%$ & $\mathbf{0 . 0 0 8}$ \\
Biopsies (cells $\left./ \mathrm{mm}^{2}\right)$ & & & \\
Total inflammatory cells & $1773(1256-2626)$ & $1045(760-1317)$ & $\mathbf{0 . 0 0 8}$ \\
Neutrophils & $13(4-26), 1 \%$ & $0(0-31), 0 \%$ & 0.100 \\
T lymphocytes & $786(648-1364), 54 \%$ & $624(439-788), 63 \%$ & $\mathbf{0 . 0 3 5}$ \\
B lymphocytes & $125(52-259), 9 \%$ & $62(28-179), 6 \%$ & 0.100 \\
Macrophages & $487(235-793), 33 \%$ & $255(102-446), 26 \%$ & $\mathbf{0 . 0 1 7}$ \\
Eosinophils & $0(0-0), 0 \%$ & $5(0-12), 0 \%$ & 0.279 \\
Mast cells & $40(14-78), 3 \%$ & $52(26-82), 5 \%$ & 0.641 \\
\hline
\end{tabular}

BAL cell counts and differentials available from 33 children with CF and 10 controls; biopsy cell counts available from 39-42 children with CF and from 13-16 controls, depending on cell type analysed; median (IQR) cell counts and percentage of total inflammatory cells shown.

\section{Pathogenic organisms: distinct associations with luminal and mucosal inflammation}

Pathogenic organisms were identified in 26 (56\%) of the 46 children with CF (table 1). The most frequent pathogens were Aspergillus spp $(\mathrm{n}=13)$ and Pseudomonas aeruginosa $(\mathrm{n}=11)$. In one child both Aspergillus spp and $P$ aeruginosa were identified.

The presence of Aspergillus spp was associated with higher numbers of BAL fluid inflammatory cells (median (IOR) 2898 $(1377-6737)$ vs $\left.1098(670-2193) \times 10^{3} / \mathrm{ml}, \mathrm{p}=0.014\right)$ and neutrophils (median (IOR) 1190 (817-5473) vs 387 (199-990) $\left.\times 10^{3} / \mathrm{ml}, \mathrm{p}=0.010\right)$. However, after adjustment for age, this association was lost ( $p=0.251$ and $p=0.235$, respectively). No differences were seen in biopsy tissue.

The presence of $P$ aeruginosa was associated with higher numbers of bronchial mucosal inflammatory cells (median (IOR)

\section{A}

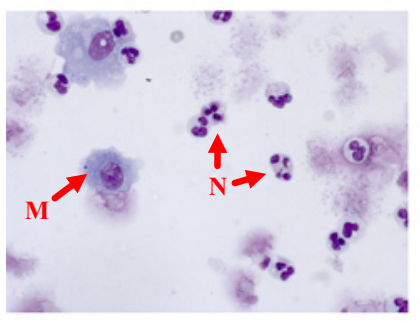

B

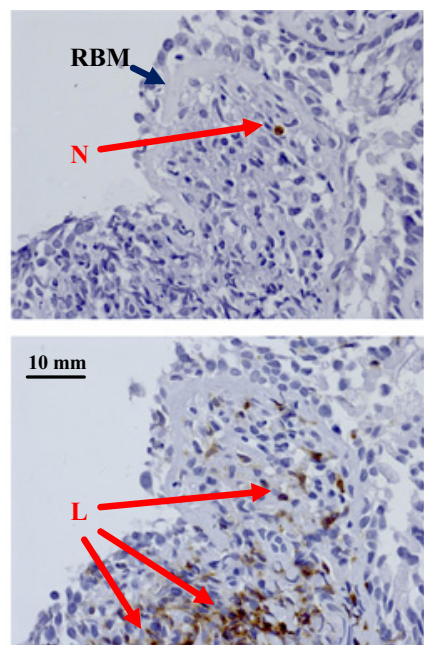

Figure 1 Representative high power view of a BAL sample obtained from a 10-year old CF child showing neutrophilic infiltration ( $A$; MayGrünwald Giemsa, original $\times 400$ ) and of an endobronchial biopsy sample obtained from the same child showing dense lymphocytic infiltration with only few neutrophils in subepithelial tissue (B: top image: anti-NE staining for neutrophils; bottom image: anti-CD3 staining for T-lymphocytes, original $\times 400$ ). $L$, lymphocyte; $M$, macrophage; $N$, neutrophils; RBM, reticular basement membrane.

$2579(1813-2723)$ vs 1335 (1165-2084) cells $\left./ \mathrm{mm}^{2}, \mathrm{p}=0.019\right)$, but here lymphocytes predominated (median (IOR) 1606 (861-2087) vs $918(720-1423)$ cells $\left./ \mathrm{mm}^{2}, \mathrm{p}=0.036\right)$ and these were of the $\mathrm{T}$ lymphocyte subset (median (IOR) 1174 (812-1936) vs 714 (575-1198) cells $\left./ \mathrm{mm}^{2}, \mathrm{p}=0.029\right)$. These differences remained significant after adjustment for age. In contrast, no differences were seen in BAL fluid.

Inflammatory cell counts in BAL fluid or biopsies were unrelated to the presence of other pathogens.

\section{Relationship of airway inflammation and markers of airway remodelling}

As we have previously reported in a smaller CF group including some of these cases, ${ }^{13}$ RBM was thicker in children with CF than in controls (mean (SD) 4.8 (1.3) $\mu \mathrm{m}$ vs 3.9 (1.0) $\mu \mathrm{m}$, $\mathrm{p}=0.008$; figure $\mathrm{E} 1$ in online supplement). Within the CF group, RBM thickness was related to age $(r=0.62, p<0.0001$; figure E2 in online supplement), as shown in children without pulmonary disease. $^{27}$ After adjustment for age in a multivariable analysis, inflammatory cell counts in BAL fluid or biopsies were unrelated to RBM thickness (data not shown).

As also reported in a smaller group including some of these cases, ${ }^{19}$ the volume fraction of ASM in bronchial tissue was larger in children with CF compared with controls (median (IOR) 0.11 $(0.06-0.17)$ vs $0.06(0.02-0.08), p=0.008$; figure $\mathrm{E} 4$ in online supplement). Inflammatory cell counts in BAL fluid or biopsies were unrelated to the ASM volume fraction (data not shown).

\section{DISCUSSION}

Because of ease of access, most of the studies on inflammation and airway remodelling in CF have been performed on BAL fluid. $^{28} 29$ In contrast, the airway wall-the site of the destructive changes-has thus far been underinvestigated. This has been mainly due to difficulties in obtaining adequate material and also possibly to an assumption that the patterns of inflammation in the CF lumen provide a complete and reliable picture of those occurring in the airway wall per se. Recent advances in bronchoscopic techniques have allowed safe investigation of inflammatory and structural changes to the airway wall in CF, even in young children and infants. ${ }^{18} 3031$ In this study we used both BAL fluid and endobronchial biopsy to study inflammation in the airway lumen and the airway wall in children with CF as young as 2 months of age in order to 
Table 3 Total and differential inflammatory cell counts in bronchoalveolar lavage (BAL) fluid and in subepithelial bronchial tissue obtained from children with cystic fibrosis (CF) during chest exacerbation ${ }^{26}$ $(n=36)$ or while well $(n=10)$

\begin{tabular}{lllr}
\hline & Exacerbation & Well & p Value \\
\hline BAL (cells $\times 10^{3}$ per ml) & & & \\
Total inflammatory cells & $1914(1098-3199)$ & $512(119-588)$ & $<\mathbf{0 . 0 0 1}$ \\
$\quad$ Neutrophils & $970(287-1584), 51 \%$ & $222(21-380), 56 \%$ & $\mathbf{0 . 0 0 4}$ \\
Lymphocytes & $49(18-150), 3 \%$ & $10(5-21), 3 \%$ & $\mathbf{0 . 0 1 9}$ \\
Macrophages & $884(433-1158), 46 \%$ & $164(94-319), 41 \%$ & $<\mathbf{0 . 0 0 1}$ \\
$\quad$ Eosinophils & $17(5-52), 1 \%$ & $1(0-3), 0 \%$ & $\mathbf{0 . 0 0 7}$ \\
Biopsies (cells/mm $\left.{ }^{2}\right)$ & & & \\
Total inflammatory cells & $1864(1296-2634)$ & $1263(742-1549)$ & 0.067 \\
Neutrophils & $14(5-23), 1 \%$ & $15(0-31), 1 \%$ & 0.964 \\
T lymphocytes & $912(693-1411), 55 \%$ & $563(398-963), 59 \%$ & $\mathbf{0 . 0 3 4}$ \\
B lymphocytes & $125(66-197), 7 \%$ & $102(38-288), 11 \%$ & 0.931 \\
Macrophages & $570(316-881), 34 \%$ & $187(56-473), 20 \%$ & $\mathbf{0 . 0 0 5}$ \\
Eosinophils & $0(0-10), 0 \%$ & $0(0-4), 0 \%$ & 0.215 \\
Mast cells & $45(21-73), 3 \%$ & $82(15-178), 9 \%$ & 0.379 \\
\hline
\end{tabular}

BAL cell counts and differentials available from 27 children with CF during chest exacerbations and from six children with CF while well; biopsy cell counts available from 29-32 children with CF during chest exacerbations and from $8-10$ children with CF while well, depending on cell type analysed; median (IQR) cell counts and percentage of total inflammatory cells shown.

*This significance was lost after adjustment for age $(p=0.227)$.

compare the two. We demonstrate that inflammation is present in both compartments early in the course of the disease and that, as hypothesised, the patterns of inflammation in the lumen and bronchial mucosa are different. In contrast to the neutrophil-dominated inflammation in the airway lumen, CF is characterised by a lymphocytic inflammation of the bronchial mucosa. Furthermore, inflammatory associations with periods of exacerbation or CF-specific pathogens also differ at these two sites. These data demonstrate that BAL fluid and endobronchial biopsy provide different but complementary information, and both need to be sampled in order to be able to understand fully the pathophysiological processes in CF.

Our finding of the presence of large numbers of neutrophils in the BAL fluid is as expected and in agreement with multiple previous studies, ${ }^{5632}$ whereas the scarcity of neutrophils in the bronchial mucosa sampled by biopsy is novel. Our interpretation is that there is likely to be an intensive neutrophil recruitment from the bronchial vasculature followed by rapid migration through the bronchial wall with their accumulation in the airway lumen where they can be identified in high numbers in BAL fluid. Alternatively, neutrophils may enter the airway luminal compartment via tissue migration occurring more distally in the smaller membranous bronchioli. The data of Hubeau et al, however, who investigated the inflammatory infiltrate of the CF bronchial mucosa in transplantation tissue, would argue against this last speculation. The neutrophils identified in their study were particularly numerous at the segmental level compared with the lobar or distal level of the bronchial tree. $^{4}$

Previous studies have described a lymphocytic infiltrate consisting mainly of $\mathrm{T}$ lymphocytes in the CF bronchial mucosa in autopsy or explant tissues recovered at end-stage disease. ${ }^{3-5}$ Our findings in biopsy tissue of higher numbers of lymphocytes in the bronchial mucosa of children indicate clearly that their presence is not just a feature of end-stage disease but part of the ongoing inflammatory process of CF. Indeed, enhanced chemotaxis of CF compared with control lymphocytes towards interleukin 8, a predominant chemokine found in BAL fluid of subjects with $\mathrm{CF}^{33}$ has recently been shown in an in vitro setting. ${ }^{34}$ The pathophysiological function of these infiltrating lymphocytes is unclear, but it could be speculated that $\mathrm{T}$ lymphocytes, the numbers of which we found to be higher during exacerbations and in the presence of $P$ aeruginosa, may be involved in disease progression-for instance, through the induction of matrix metalloproteinase expression, known to be present in BAL fluid of subjects with CF and believed to be involved in airway tissue destruction. ${ }^{35} 36$ In the study by Hubeau et al there were more T lymphocytes, particularly at the distal level of the bronchial tree where intense tissue damage was observed. ${ }^{4}$ We are currently investigating further their role in early CF lung disease. ${ }^{20}$

Attention has recently turned to the role of alveolar macrophages in progression of CF lung disease. ${ }^{37} 38$ Brennan et al reported higher absolute macrophage numbers but lower percentages in BAL fluid obtained from preschool children with CF with evidence of bacterial infection compared with uninfected children, suggesting that relatively fewer macrophages present during a pulmonary infection may have a diminished ability to phagocytise the increasing population of dying neutrophils. ${ }^{38}$ Our findings of both higher macrophage numbers and percentages in the airway lumen and mucosa during CF exacerbations would argue against this hypothesis. The contribution of these cells to the disease process requires further investigation, but data obtained from CF fetal tissue suggest their possible involvement in the early onset of inflammation in infants with CF. ${ }^{39}$

We observed that chest exacerbations manifest differently in the bronchial lumen (higher numbers of all inflammatory cell types) and in the mucosa (higher numbers of lymphocytes and macrophages, but not of neutrophils). The higher numbers of submucosal lymphocytes during exacerbations are reminiscent of observations made in mild asthma exacerbations, ${ }^{40}$ but the lack of higher numbers of submucosal neutrophils contrasts with findings reported in exacerbations of severe asthma ${ }^{41}$ and chronic obstructive pulmonary disease, ${ }^{42}$ suggesting that mechanisms of exacerbation are disease-specific. It could be speculated that the main source of neutrophil chemoattractants in the CF lung derives from the airway lumen (eg, soluble bacterial products, complement fragments or substances produced by airway luminal cellular components) or from the apical part of the airway epithelium ${ }^{33} 34$ whereas, in other chronic airway diseases, neutrophil chemoattractants such as 
CXCL8 (interleukin 8) or CXCL5 (ENA-78) originate from the submucosa. ${ }^{41} 42$ We also found that different organisms had differential associations with luminal and mucosal inflammation. While the presence of $P$ aeruginosa was associated with higher numbers of lymphocytes in the bronchial mucosa, no cell count differences between Pseudomonas-positive and Pseudomonas-negative patients were seen in BAL fluid, suggesting a role for submucosal lymphocytes in host defence against this pathogen as shown in animal models. ${ }^{43}{ }^{44}$ Chronic infection with $P$ aeruginosa has clear detrimental effects on lung function and prognosis, perhaps underscoring the role of submucosal lymphocytic inflammation in disease progression. In contrast, we observed higher numbers of neutrophils in BAL fluid in the presence of Aspergillus spp, but no cell count differences between Aspergillus-positive and Aspergillus-negative patients were seen in the biopsies. However, the association in BAL fluid was lost after correction for age, suggesting that the presence of Aspergillus spp in BAL fluid is rather a marker of more severe inflammation and advanced or prolonged lung disease (ie, older age) than independently associated with disease progression, as recently proposed. ${ }^{45}$ In our study we could not differentiate between colonisation and infection with pathogens; further work is needed to clarify their relationship with both luminal and mucosal inflammation.

\section{Study limitations}

We acknowledge that one reason for the lack of correlation of cells in BAL fluid with cells in biopsies could be technical, as endobronchial biopsy and BAL sample different areas of the airways. Endobronchial biopsy samples come from relatively proximal large airways and the airway mucosa of bronchial bifurcations (carinae) and they do not represent the full airway wall thickness. Our data may therefore not be representative of the entirety of the conducting airways, and we may have missed changes occurring in subsegmental airways and therefore associations with, for example, spirometry, even though spirometry is probably too insensitive to detect subtle changes. ${ }^{46}$ Endobronchial biopsies are further limited in size and there is considerable variability between samples of a given individual, so multiple biopsies per subject should ideally be analysed. ${ }^{47}{ }^{48}$ Endobronchial biopsy in children is challenging, ${ }^{18} 31$ and we were only able to analyse $1-2$ biopsies of adequate morphology per child. Although our study was powered sufficiently to detect between-group differences, ${ }^{24}$ some relationships within individuals may thus have been missed due to inadequate sampling. The findings of our study need to be interpreted in light of these limitations. However, the strength of our data lies in the relatively large number of biopsies studied and in the inclusion of disease-free controls.

\section{Conclusions}

Our data show that inflammation is present in both the airway lumen and the bronchial mucosa but that, in support of our hypothesis, the patterns of inflammation and the responses to infective stimuli observed in BAL fluid and biopsies obtained from children with CF are dissimilar. In contrast to the neutrophil-dominated inflammation present in the airway lumen, CF is characterised by a lymphocytic infiltration and accumulation of the bronchial mucosa, the function of which merits further investigation. BAL and endobronchial biopsy provide different but complementary information, underscoring the need to apply both techniques in the study of the pathophysiological processes in the CF airway. Considering that progressive airway wall changes ultimately lead to bronchiec- tasis, we suggest that further research should focus on understanding the pathogenetic mechanisms at this tissue site.

Acknowledgments We thank Chloe Dunn, Bernie Ortega, Carmen Lacruz, Eleanor Singh, Gemma Moody and the staff of the Department of Anaesthesia, Royal Brompton Hospital, for their assistance with bronchoscopies; Mark Rosenthal and lan Balfour-Lynn for performing some of the bronchoscopies; Nikki Cornish for excellent technical assistance; and Andrew Nicholson and the Department of Pathology, Royal Brompton Hospital for their preparation of biopsy material. We also gratefully acknowledge the patients and families who agreed to take part in the study.

Funding NR is the recipient of a European Respiratory Society Fellowship (Nr. 64) and of a grant from the Swiss National Science Foundation (SSMBS Nr. 1172).

Competing interests None.

Patient consent Obtained

Ethics approval Ethics approval was provided by Royal Brompton Harefield and NHLI ethics committee.

Contributors Conception and design of the study: NR, TNH, EWFWA, PKJ, AB, JCD. Acquisition of data: NR, LT, TNH, HT, JZ, Y-SQ, AB, JCD. Analysis and interpretation of data: NR, OF, EWFWA, PKJ, AB, JCD. Drafting and revising: NR, OF, PKJ, AB, JCD. Important intellectual content: NR, TNH, EWFWA, PKJ, AB, JCD. Final approval: all authors.

Provenance and peer review Not commissioned; externally peer reviewed.

\section{REFERENCES}

1. O'Sullivan BP, Freedman SD. Cystic fibrosis. Lancet 2009;373:1891-904.

2. Lex C, Ferreira F, Zacharasiewicz A, et al. Airway eosinophilia in children with severe asthma: predictive values of noninvasive tests. Am J Respir Crit Care Med 2006;174:1286-91.

3. Azzawi M, Johnston PW, Majumdar S, et al. T lymphocytes and activated eosinophils in airway mucosa in fatal asthma and cystic fibrosis. Am Rev Respir Dis 1992; 145:1477-82

4. Hubeau C, Lorenzato M, Couetil JP, et al. Quantitative analysis of inflammatory cells infiltrating the cystic fibrosis airway mucosa. Clin Exp Immunol 2001;124:69-76.

5. Hamutcu R, Rowland JM, Horn MV, et al. Clinical findings and lung pathology in children with cystic fibrosis. Am J Respir Crit Care Med 2002:165:1172-5.

6. Sly PD, Brennan S, Gangell C, et al; Australian Respiratory Early Surveillance Team for Cystic Fibrosis (AREST-CF). Lung disease at diagnosis in infants with cystic fibrosis detected by newborn screening. Am J Respir Crit Care Med 2009; 180:146-52.

7. Long $\mathbf{F R}$, Williams RS, Castile RG. Structural airway abnormalities in infants and young children with cystic fibrosis. J Pediatr 2004;144:154-61.

8. Martinez TM, Llapur CJ, Williams TH, et al. High-resolution computed tomography imaging of airway disease in infants with cystic fibrosis. Am J Respir Crit Care Med 2005:172:1133-8.

9. Stick SM, Brennan S, Murray C, et al; Australian Respiratory Early Surveillance Team for Cystic Fibrosis (AREST CF). Bronchiectasis in infants and preschool children diagnosed with cystic fibrosis after newborn screening. J Pediatr 2009;155:623-8.e1.

10. Ranganathan SC, Stocks J, Dezateux C, et al. The evolution of airway function in early childhood following clinical diagnosis of cystic fibrosis. Am J Respir Crit Care Med 2004;169:928-33

11. Linnane BM, Hall GL, Nolan G, et al; AREST-CF. Lung function in infants with cystic fibrosis diagnosed by newborn screening. Am J Respir Crit Care Med 2008;178:1238-44

12. Regamey N, Jeffery PK, Alton EW, et al. Airway remodelling and its relationship to inflammation in cystic fibrosis. Thorax 2011;66:624-9.

13. Hilliard TN, Regamey N, Shute JK, et al. Airway remodelling in children with cystic fibrosis. Thorax 2007;62:1074-80.

14. Hilliard TN, Zhu J, Farley R, et al. Nasal abnormalities in cystic fibrosis mice independent of infection and inflammation. Am J Respir Cell Mol Biol 2008;39:19-25.

15. Farrell PM, Rosenstein BJ, White TB, et al; Cystic Fibrosis Foundation. Guidelines for diagnosis of cystic fibrosis in newborns through older adults: Cystic Fibrosis Foundation consensus report. J Pediatr 2008;153:S4-14.

16. de Blic J, Midulla F, Barbato A, et al. Bronchoalveolar lavage in children. ERS Task Force on bronchoalveolar lavage in children. European Respiratory Society. Eur Respir J 2000;15:217-31.

17. Payne DN, Oiu Y, Zhu J, et al. Airway inflammation in children with difficult asthma: relationships with airflow limitation and persistent symptoms. Thorax 2004;59:862-9.

18. Regamey N, Hilliard TN, Saglani S, et al. Quality, size, and composition of pediatric endobronchial biopsies in cystic fibrosis. Chest 2007;131:1710-17.

19. Regamey N, Ochs M, Hilliard TN, et al. Increased airway smooth muscle mass in children with asthma, cystic fibrosis, and non-cystic fibrosis bronchiectasis. Am J Respir Crit Care Med 2008;177:837-43. 
20. Tan HL, Regamey N, Brown S, et al. The Th17 pathway in cystic fibrosis lung disease. Am J Respir Crit Care Med 2011;184:252-8.

21. Barbato A, Turato G, Baraldo $\mathrm{S}$, et al. Epithelial damage and angiogenesis in the airways of children with asthma. Am J Respir Crit Care Med 2006:174:975-81.

22. Tsoumakidou M, Elston W, Zhu J, et al. Cigarette smoking alters bronchial mucosal immunity in asthma. Am J Respir Crit Care Med 2007:175:919-25.

23. Sullivan $\mathbf{P}$, Stephens D, Ansari T, et al. Variation in the measurements of basement membrane thickness and inflammatory cell number in bronchial biopsies. Eur Respir $\mathrm{J}$ 1998:12:811-15.

24. Sont JK, Willems LN, Evertse CE, et al. Repeatability of measures of inflammatory cell number in bronchial biopsies in atopic asthma. Eur Respir J 1997;10:2602-8.

25. Altman D. Practical Statistics for Medical Research. London: Chapman \& Hall, 1991.

26. Ferkol T, Rosenfeld M, Milla CE. Cystic fibrosis pulmonary exacerbations. J Pediatr 2006;148:259-64.

27. Tsartsali L, Hislop AA, McKay K, et al. Development of the bronchial epithelia reticular basement membrane: relationship to epithelial height and age. Thorax 2011:66:280-5

28. Birrer P, McElvaney NG, Rudeberg A, et al. Protease-antiprotease imbalance in the lungs of children with cystic fibrosis. Am J Respir Crit Care Med 1994;150:207-13.

29. Bruce MC, Poncz L, Klinger JD, et al. Biochemical and pathologic evidence for proteolytic destruction of lung connective tissue in cystic fibrosis. Am Rev Respir Dis 1985; 132:529-35

30. Molina-Teran A, Hilliard TN, Saglani S, et al. Safety of endobronchial biopsy in children with cystic fibrosis. Pediatr Pulmonol 2006;41:1021-4.

31. Regamey N, Balfour-Lynn I, Rosenthal $\mathrm{M}$, et al. Time required to obtain endobronchial biopsies in children during fiberoptic bronchoscopy. Pediatr Pulmonol 2009;44:76-9

32. Bedrossian CW, Greenberg SD, Singer DB, et al. The lung in cystic fibrosis. A quantitative study including prevalence of pathologic findings among different age groups. Hum Pathol 1976; 7:195-204.

33. De Rose V. Mechanisms and markers of airway inflammation in cystic fibrosis. Eur Respir J 2002;19:333-40.

34. Al Alam D, Deslee G, Tournois C, et al. Impaired interleukin-8 chemokine secretion by staphylococcus aureus-activated epithelium and T-cell chemotaxis in cystic fibrosis. Am J Respir Cell Mol Biol 2010;42:644-50.

35. Prause 0, Bozinovski S, Anderson GP, et al. Increased matrix metalloproteinase- 9 concentration and activity after stimulation with interleukin-17 in mouse airways. Thorax 2004;59:313-17.
36. Zheng L, Lam WK, Tipoe GL, et al. Overexpression of matrix metalloproteinase-8 and -9 in bronchiectatic airways in vivo. Eur Respir J 2002;20:170-6.

37. Bruscia EM, Zhang PX, Ferreira E, et al. Macrophages directly contribute to the exaggerated inflammatory response in cystic fibrosis transmembrane conductance regulator-/- mice. Am J Respir Cell Mol Biol 2009;40:295-304.

38. Brennan S, Sly PD, Gangell CL, et al. Alveolar macrophages and CC chemokines are increased in children with cystic fibrosis. Eur Respir J 2009;34:655-61.

39. Hubeau C, Puchelle E, Gaillard D. Distinct pattern of immune cell population in the lung of human fetuses with cystic fibrosis. J Allergy Clin Immunol 2001;108:524-9.

40. Castro M, Bloch SR, Jenkerson MV, et al. Asthma exacerbations after glucocorticoid withdrawal reflects T cell recruitment to the airway. Am J Respir Crit Care Med 2004:169:842-9

41. Qiu Y, Zhu J, Bandi V, et al. Bronchial mucosal inflammation and upregulation of CXC chemoattractants and receptors in severe exacerbations of asthma. Thorax 2007:62:475-82

42. Oiu Y, Zhu J, Bandi V, et al. Biopsy neutrophilia, neutrophil chemokine and receptor gene expression in severe exacerbations of chronic obstructive pulmonary disease. Am J Respir Crit Care Med 2003;168:968-75.

43. Dubin PJ, Kolls JK. IL-23 mediates inflammatory responses to mucoid Pseudomonas aeruginosa lung infection in mice. Am J Physiol Lung Cell Mol Physio 2007;292:L519-28.

44. Liu J, Feng Y, Yang K, et al. Early production of IL-17 protects against acute pulmonary Pseudomonas aeruginosa infection in mice. FEMS Immunol Med Microbiol 2011;61:179-88

45. Amin R, Dupuis A, Aaron SD, et al. The effect of chronic infection with Aspergillus fumigatus on lung function and hospitalization in patients with cystic fibrosis. Ches 2010;137:171-6.

46. Kraemer R, Blum A, Schibler A, et al. Ventilation inhomogeneities in relation to standard lung function in patients with cystic fibrosis. Am J Respir Crit Care Med 2005:171:371-8.

47. Gamble E, Qiu $Y$, Wang D, et al. Variability of bronchial inflammation in chronic obstructive pulmonary disease: implications for study design. Eur Respir $J$ 2006:27:293-9.

48. Jeffery P, Holgate S, Wenzel S; Endobronchial Biopsy Workshop. Methods for the assessment of endobronchial biopsies in clinical research: application to studies of pathogenesis and the effects of treatment. Am J Respir Crit Care Med 2003;168:S1-17

\section{Journal club}

\section{Role of kinase suppressor of Ras-1 in Pseudomonas aeruginosa infections}

Respiratory infection with Pseudomonas aeruginosa can have serious implications, particularly on a background of immunodeficiency, cystic fibrosis and mechanical ventilation. In this study, by conducting a series of experiments on mice, the authors identified the key role of the kinase suppressor of Ras-1 (Ksr1), an enzymatic protein, in the innate host response to P aeruginosa infection.

Ksr1 deficiency impairs the bactericidal activity of alveolar macrophages and, as a consequence, Ksr1-deficient mice were found to die of sepsis from failed clearance of $P$ aeruginosa. The bactericidal activity of alveolar macrophages and neutrophils is mediated by the formation and release of nitric oxide (NO) and peroxynitrite, which is triggered by Ksr1. This occurs through a previously unidentified pathway where Ksr1 functions as a unique scaffold and mediates the interaction between inducible NO synthase (iNOS) and heat shock protein 90 , thereby activating iNOS and releasing NO, which kills the bacteria.

The authors concluded that this study identifies a unique role of Ksr1 in bacterial infection and they have shown a link between Ksr1 and the regulation of bacterial pneumonia and sepsis.

Zhang Y, Li X, Carpinteiro A, et al. Kinase suppressor of Ras-1 protects against pulmonary Pseudomonas aeruginosa infections. Nat Med 2003;17:341-6.

\section{Syed Huq}

Correspondence to Dr Syed Huq, ST5 Respiratory Medicine, Liverpool Heart and Chest Hospital, Liverpool, UK; syedhuq@nhs.net 